\title{
Finite-Time Stability and Controller Design of Continuous-Time Polynomial Fuzzy Systems
}

\author{
Xiaoxing Chen and Manfeng Hu \\ School of Science, Jiangnan University, Wuxi 214122, China \\ Correspondence should be addressed to Manfeng Hu; humanfeng@jiangnan.edu.cn
}

Received 9 September 2017; Revised 28 October 2017; Accepted 12 November 2017; Published 24 December 2017

Academic Editor: Valery Y. Glizer

Copyright (C) 2017 Xiaoxing Chen and Manfeng Hu. This is an open access article distributed under the Creative Commons Attribution License, which permits unrestricted use, distribution, and reproduction in any medium, provided the original work is properly cited.

\begin{abstract}
Finite-time stability and stabilization problem is first investigated for continuous-time polynomial fuzzy systems. The concept of finite-time stability and stabilization is given for polynomial fuzzy systems based on the idea of classical references. A sumof-squares- (SOS-) based approach is used to obtain the finite-time stability and stabilization conditions, which include some classical results as special cases. The proposed conditions can be solved with the help of powerful Matlab toolbox SOSTOOLS and a semidefinite-program (SDP) solver. Finally, two numerical examples and one practical example are employed to illustrate the validity and effectiveness of the provided conditions.
\end{abstract}

\section{Introduction}

Over the past decades, the fuzzy logic control has developed into a successful and fruitful branch of automation and control theory due to the fact that the fuzzy models are the appealing and efficient tools in approximating the complex nonlinear dynamical systems. Among different fuzzy models, the well-known Takagi-Sugeno (T-S) fuzzy model has attracted considerable research attention [1-5] and a large amount of literature has appeared on the fundamental issues of stability and stabilization for T-S fuzzy systems (see, e.g., $[6,7]$ and the cited therein). Very recently, Choi et al. [8] put forward a new method to formulate a framework that can describe T-S fuzzy systems with time-varying input delay and output constraints based on $(Q, S, R)-\alpha$-dissipativity, which not only synthesized $H_{\infty}$ control and passivity control, but also applied the obtained results to different road conditions. In the field of the fuzzy controller design, the authors in [9] considered input saturation of the nonlinear systems and employed a variables separation approach and the smallgain approach to design an adaptive back-stepping fuzzy controller, which provided more extensive applications in practice.
Recently, the T-S fuzzy model has been extended to polynomial fuzzy model [10-15]. SOS approach to polynomial fuzzy system has been presented simultaneously and provides more extensive and/or relaxed results for the existing LMI approaches to T-S fuzzy system. A feasible solution to the SOS-based stability conditions can be found numerically with the help of a powerful Matlab toolbox SOSTOOLS [16].

Stability and stabilization conditions in terms of SOS are central to the problem of stability analysis and control design for polynomial fuzzy model, which have been investigated in the past ten years. For example, Tanaka et al. [10-14] firstly proposed SOS-based framework to obtain stability and stabilization conditions of the polynomial fuzzy systems based on polynomial Lyapunov functions. Recently, a new SOS design framework for robust control of polynomial fuzzy systems with uncertainties is presented by the research group of professor Tanaka [17]. During this period, professor Lam and his colleagues make great contributions to relax the stability and stabilization conditions. For instance, shapedependent SOS-based stability analysis of the polynomial fuzzy-control systems is investigated in [18]. A piecewiselinear membership function [19] and a switching polynomial Lyapunov function [20] are separately proposed to facilitate 
the stability analysis. Some other related issues of the polynomial fuzzy model have also been investigated for closed-form estimates of the domain of attraction [21], for tracking control of polynomial fuzzy networked systems [22], and for stability analysis and controller design of discrete-time polynomial fuzzy time-varying delay systems [23].

As is well known to the control community, the behavior of a system over a fixed time interval is practicable and then widely concerned. In view of this, a concept of finite-time stability (FTS) is introduced in $[24,25]$ and great attention has been paid to (T-S) fuzzy model [26-29]. However, the finitetime stability analysis and controller design of polynomial fuzzy model are seldom discussed.

Motivated by above discussions, in this paper, the problem of finite-time stability and stabilization for a class of continuous-time polynomial fuzzy system is considered. The main contributions of this paper are twofold: (1) the concept of finite-time stability for polynomial fuzzy model is firstly proposed, and (2) finite-time stabilizing controller is designed to stabilize the nonlinear system represented in the form of SOS, which is superior to the form of LMIs.

The rest of this paper is organized as follows: some foundational descriptions, necessary definitions, and relevant lemmas are recalled in Section 2. The main results are obtained in Section 3. Firstly, finite-time stability conditions for the polynomial fuzzy system without any control input are derived by using a new polynomial Lyapunov-Krasovskii functional. Secondly, the stability conditions are extended to finite-time stabilization conditions for the continuous-time polynomial fuzzy system with a controller in terms of SOS. All conditions can be solved via the SOSTOOLS and an SDP solver. Section 4 provides three illustrative examples. Finally, conclusions are drawn in Section 5.

Notations. Throughout this paper, the notations employed are fairly standard. The superscripts -1 and $T$ stand for the inverse and transpose of a matrix, respectively. $P>0(P \geq 0$, $P<0$, and $P \leq 0$ ) means that the matrix $P$ is positive definite (positive semidefinite, negative definite, and negative semidefinite). $R^{m \times n}$ is the set of $m \times n$ real matrices. $I$ denotes the identity matrix with compatible dimensions.

\section{Preliminaries and Problem Formulation}

Consider the following nonlinear system:

$$
\dot{x}(t)=f(x(t), u(t)),
$$

where $f$ is a nonlinear function, $x(t)=$ $\left[\begin{array}{llll}x_{1}(t) & x_{2}(t) & \cdots & x_{n}(t)\end{array}\right]^{T}$ is the state vector, and $u(t)=$ $\left[\begin{array}{llll}u_{1}(t) & u_{2}(t) & \cdots & u_{m}(t)\end{array}\right]^{T}$ is the input vector. Based on the sector nonlinearity concept [1], system (1) can be expressed in the following polynomial if-then rules.

Model Rule i. If $z_{1}(t)$ is $\mathscr{M}_{i 1}$ and $z_{2}(t)$ is $\mathscr{M}_{i 2}$ and $\ldots$ and $z_{p}(t)$ is $\mathscr{M}_{i p}$, then

$$
\dot{x}(t)=A_{i}(x(t)) \hat{x}(x(t))+B_{i}(x(t)) u(t),
$$

where $A_{i}(x(t)) \in R^{n \times n}$ and $B_{i}(x(t)) \in R^{n \times m}(i=1,2, \ldots, r$; $r$ is the number of if-then rules.) are polynomial matrices in $x(t)$. The term $\widehat{x}(x(t)) \in R^{N}$ is an $N \times 1$ column vector whose entries are all monomials in $x(t)$.

The defuzzification process of model (2) can be calculated as below (for more details, see [10]):

$$
\begin{aligned}
\dot{x}(t) & \\
= & \frac{\sum_{i=1}^{r} \omega_{i}(z(t))\left\{A_{i}(x(t)) \hat{x}(x(t))+B_{i}(x(t)) u(t)\right\}}{\sum_{i=1}^{r} \omega_{i}(z(t))} \\
& =\sum_{i=1}^{r} h_{i}(z(t))\left\{A_{i}(x(t)) \hat{x}(x(t))+B_{i}(x(t)) u(t)\right\},
\end{aligned}
$$

where

$$
\begin{aligned}
z(t) & =\left[\begin{array}{lll}
z_{1}(t) & \cdots & z_{p}(t)
\end{array}\right], \\
\omega_{i}(z(t)) & =\prod_{j=1}^{p} M_{i j}\left(z_{j}(t)\right), \\
\sum_{i=1}^{r} \omega_{i}(z(t)) & >0, \quad \omega_{i}(z(t)) \geq 0, i=1,2, \ldots, r, \\
h_{i}(z(t)) & \triangleq \frac{\omega_{i}(z(t))}{\sum_{i=1}^{r} \omega_{i}(z(t))} \geq 0, \\
\sum_{i=1}^{r} h_{i}(z(t)) & =1 .
\end{aligned}
$$

Before proceeding, the following definitions and lemma are necessary.

Definition 1 (see [16]). A polynomial $p(x), x \in R^{N}$, is an SOS, if there exist polynomials $f_{1}(x), \ldots, f_{m}(x)$ such that

$$
p(x)=\sum_{i=1}^{m} f_{i}^{2}(x) .
$$

Definition 2 (see [16]). The SOS condition (5) is equivalent to the existence of a positive semidefinite matrix $Q$, such that

$$
p(x)=Z^{T}(x) Q Z(x),
$$

where $Z(x)$ is some properly chosen vector of monomials.

Definition 3 (finite-time stability). Given a positive definite matrix $R$ and three positive constants $c_{1}, c_{2}, T_{f}\left(c_{1}<c_{2}\right)$, the polynomial fuzzy system (3) with $u(t) \equiv 0$ is said to be finite-time stable with respect to $\left(c_{1}, c_{2}, T_{f}, R\right)$, if $\widehat{x}^{T}(x(0)) R \widehat{x}(x(0))<c_{1} \Rightarrow \widehat{x}^{T}(x(t)) R \widehat{x}(x(t))<c_{2}, \forall t \in$ $\left[0, T_{f}\right]$.

Definition 4 (finite-time stabilization). Given a positive definite matrix $R$ and three positive constants $c_{1}, c_{2}, T_{f}\left(c_{1}<c_{2}\right)$, the polynomial fuzzy system (3) with control input $u(t)$ is said to be finite-time stabilization with respect to $\left(c_{1}, c_{2}, T_{f}, R\right)$, if $\widehat{x}^{T}(x(0)) R \widehat{x}(x(0))<c_{1} \Rightarrow \widehat{x}^{T}(x(t)) R \widehat{x}(x(t))<c_{2}, \forall t \in$ $\left[0, T_{f}\right]$. 
Remark 5. Definitions 3 and 4 are a first attempt to give the concept of finite-time stability and stabilization for polynomial fuzzy systems based on the basic idea in classical paper $[24,25]$. When $\widehat{x}(x(t))$ here reduces to $x(t)$, the above two definitions reduce to the forms in [24].

Lemma 6 (see [30]). Let $F(x(t))$ be an $N \times N$ symmetric polynomial matrix of degree $2 d$ in $x(t) \in R^{n}$. Furthermore, let $\widehat{x}(x(t))$ be a column vector whose entries are all monomials in $x(t)$ with degree not greater than $d$. If $v^{T}(t) F(x(t)) v(t)$ is an SOS, where $v(t) \in R^{N}$, then $F(x(t)) \geq 0$ for all $x(t) \in R^{n}$.

\section{Main Results}

In this section, the finite-time stability and stabilization conditions for the continuous-time polynomial fuzzy system (3) are given, respectively.

3.1. Finite-Time Stability of Continuous-Time Polynomial Fuzzy System. Firstly, we will analyze the finite-time stability of the continuous-time polynomial fuzzy system (3) with $u(t)=0$. We drop the notation with respect to time $t$. For example, we will use $x, \widehat{x}(x)$ to replace $x(t), \widehat{x}(x(t))$, respectively $[10,11]$. In addition, $A_{i}^{k}(x)$ refers to the $k$ th row of $A_{i}(x)$. Then system (3) with $u=0$ has the following form:

$$
\dot{x}=\sum_{i=1}^{r} h_{i}(z(t)) A_{i}(x) \widehat{x}(x) .
$$

Theorem 7. Suppose there exist a symmetric polynomial matrix $P(x) \in R^{N \times N}$, a constant $\alpha \geq 0$, and two positive scalars $\Phi_{1}$ and $\Phi_{2}$ such that the following are satisfied:

$$
\begin{aligned}
& \hat{x}^{T}(x)\left(P(x)-\epsilon_{1}(x) I\right) \widehat{x}(x) \text { is SOS, } \\
& -\hat{x}^{T}(x)\left(P(x) T(x) A_{i}(x)+A_{i}^{T}(x) T^{T}(x) P(x)\right. \\
& \left.\quad+\sum_{k=1}^{n} \frac{\partial P(x)}{\partial x_{k}} A_{i}^{k}(x) \hat{x}(x)-\alpha P(x)+\epsilon_{2 i}(x) I\right) \hat{x}(x) \\
& \quad \text { is } S O S, \quad \forall i, \\
& \hat{x}^{T}(x)\left(P(x)-\Phi_{1} R-\epsilon_{3}(x) I\right) \hat{x}(x) \text { is SOS, } \\
& \hat{x}^{T}(x)\left(\Phi_{2} R-P(x)-\epsilon_{4}(x) I\right) \hat{x}(x) \text { is SOS, } \\
& \hat{x}^{T}(x)\left(\Phi_{1} c_{2}-\Phi_{2} c_{1} e^{\alpha T_{f}}-\epsilon_{5}(x)\right) \hat{x}(x) \text { is SOS, }
\end{aligned}
$$

where polynomials $\epsilon_{1}(x)>0, \epsilon_{2 i}(x)>0, \epsilon_{3}(x)>0, \epsilon_{4}(x)>0$, and $\epsilon_{5}(x)>0$ for $x \neq 0$ and the $(i, j)$ th entry of polynomial matrix $T(x) \in R^{N \times n}$ is given by

$$
T^{i j}(x)=\frac{\partial \widehat{x}_{i}}{\partial x_{j}}(x) ;
$$

then the continuous-time polynomial fuzzy system (3) with $u=$ 0 is finite-time stable with respect to $\left(c_{1}, c_{2}, T_{f}, R\right)$.
Proof. Choose the following candidate polynomial Lyapunov functional:

$$
V(x)=\widehat{x}^{T}(x) P(x) \widehat{x}(x) .
$$

It can be seen from condition (8) that $P(x)$ is positive definite for all $x$, and thus, $V(x)$ is a positive definite function of $x$.

The time derivation of $V(x)$ along system (7) is given by

$$
\begin{aligned}
\dot{V}(x)= & \widehat{x}^{T}(x) P(x) \dot{\hat{x}}(x)+\dot{\hat{x}}^{T}(x) P(x) \widehat{x}(x) \\
& +\widehat{x}^{T}(x) \dot{P}(x) \widehat{x}(x) \\
= & \widehat{x}^{T}(x) P(x) T(x) \dot{x}(x) \\
& +\dot{x}^{T}(x) T^{T}(x) P(x) \widehat{x}(x) \\
& +\widehat{x}^{T}(x)\left(\sum_{k=1}^{n} \frac{\partial P(x)}{\partial x_{k}} \dot{x}_{k}\right) \widehat{x}(x) .
\end{aligned}
$$

On the other hand, $\dot{x}_{k}$ can be represented as

$$
\dot{x}_{k}=\sum_{i=1}^{r} h_{i}(z) A_{i}^{k}(x) \hat{x}(x) .
$$

From (7), (15), and (16), $\dot{V}(x)$ is rewritten as

$$
\begin{aligned}
& \dot{V}(x)=\sum_{i=1}^{r} h_{i}(z) \hat{x}^{T}(x)\left(P(x) T(x) A_{i}(x)\right. \\
& \left.+A_{i}^{T}(x) T^{T}(x) P(x)+\sum_{k=1}^{n} \frac{\partial P(x)}{\partial x_{k}} A_{i}^{k}(x) \hat{x}(x)\right) \\
& \quad \cdot \hat{x}(x) .
\end{aligned}
$$

In addition, condition (9) implies that

$$
\begin{aligned}
& \widehat{x}^{T}(x)\left(P(x) T(x) A_{i}(x)+A_{i}^{T}(x) T^{T}(x) P(x)\right. \\
& \left.+\sum_{k=1}^{n} \frac{\partial P(x)}{\partial x_{k}} A_{i}^{k}(x) \widehat{x}(x)\right) \widehat{x}(x)<\widehat{x}^{T}(x)(\alpha P(x)) \\
& \quad \cdot \widehat{x}(x) .
\end{aligned}
$$

Thus, (17) yields

$$
\begin{aligned}
\dot{V}(x) & <\sum_{i=1}^{r} h_{i}(z) \widehat{x}^{T}(x)(\alpha P(x)) \widehat{x}(x) \\
& =\sum_{i=1}^{r} h_{i}(z)\left(\alpha \widehat{x}^{T}(x) P(x) \widehat{x}(x)\right)=\alpha V(x) .
\end{aligned}
$$

Integrating (19) from 0 to $t$, with $t \in\left(0, T_{f}\right]$, we have

$$
V(x)<e^{\alpha t} V(x(0)) .
$$


Along with (10) and (11), we obtain

$$
\begin{aligned}
V(x) & =\widehat{x}^{T}(x) P(x) \widehat{x}(x) \geq \widehat{x}^{T}(x) \Phi_{1} R \widehat{x}(x) \\
& =\Phi_{1} \widehat{x}^{T}(x) R \widehat{x}(x), \\
V(x(0)) & =\widehat{x}^{T}(x(0)) P(x(0)) \hat{x}(x(0)) \\
& \leq \widehat{x}^{T}(x(0)) \Phi_{2} R \widehat{x}(x(0)) \\
& =\Phi_{2} \widehat{x}^{T}(x(0)) R \hat{x}(x(0)) .
\end{aligned}
$$

Considering $\widehat{x}^{T}(x(0)) R \widehat{x}(x(0))<c_{1}$ and (20)-(21), we get

$$
\begin{aligned}
\widehat{x}^{T}(x) R \widehat{x}(x) & \leq \frac{V(x)}{\Phi_{1}}<\frac{e^{\alpha t} V(x(0))}{\Phi_{1}} \\
& \leq \frac{e^{\alpha T_{f}} V(x(0))}{\Phi_{1}} \\
& \leq \frac{e^{\alpha T_{f}} \Phi_{2} \hat{x}^{T}(x(0)) R \widehat{x}(x(0))}{\Phi_{1}} \\
& <\frac{\Phi_{2}}{\Phi_{1}} c_{1} e^{\alpha T_{f}} .
\end{aligned}
$$

Condition (12) implies that $\Phi_{2} c_{1} e^{\alpha T_{f}} \leq \Phi_{1} c_{2}$. Then

$$
\widehat{x}^{T}(x) R \widehat{x}(x)<\frac{\Phi_{2}}{\Phi_{1}} c_{1} e^{\alpha T_{f}} \leq \frac{\Phi_{1} c_{2}}{\Phi_{1}}=c_{2} .
$$

According to Definition 3, system (7) is finite-time stable with respect to $\left(c_{1}, c_{2}, T_{f}, R\right)$. That is to say, polynomial fuzzy system (3) with $u=0$ is finite-time stable with respect to $\left(c_{1}, c_{2}, T_{f}, R\right)$. This completes the proof.

Finite-time stability conditions of the continuous-time polynomial fuzzy system (3) with $u=0$, which can be checked by the SOSTOOLS [16], have been derived in Theorem 7. When $A_{i}(x)$ and $P(x)$ in Theorem 7 reduce to the constant matrices $A$ and $P$, we can get the following corollary.

Corollary 8 (see [25]). Let $\bar{P}=R^{-1 / 2} P \mathrm{R}^{-1 / 2}$ and suppose that if there exist a nonnegative scalar $\alpha$, three positive scalars $c_{1}, c_{2}, T$, with $c_{1}<c_{2}$, and a positive definite matrix $P \in R^{n \times n}$ such that

$$
\begin{aligned}
& A^{T} \bar{P}+\bar{P} A-\alpha \bar{P}<0, \\
& \frac{\lambda_{\max }(P)}{\lambda_{\min }(P)}<\frac{c_{2}}{c_{1}} e^{-\alpha T},
\end{aligned}
$$

then the linear system $\dot{x}(t)=A x(t)$ is said to be finite-time stable with respect to $\left(c_{1}, c_{2}, T, R\right)$.

Remark 9. It is well known that [25] is a classical paper in the concept of finite-time stability. As shown in Corollary 8, the SOS conditions (8)-(12) for finite-time stability in Theorem 7 reduce to the well-known LMI conditions (24). In addition, two parameters $\Phi_{1}$ and $\Phi_{2}$ used in Theorem 7 instead of the role of eigenvalues of $P$ in [25] and the condition like (25) in Corollary 8 are not needed, which help find more relaxed solutions for the range of $P(x)$ in our proposed method. Moreover, small-gain theorem in [9] is proposed to ensure the resulting closed-loop system to be bounded, but our method employs SOS approach to ensure the range of $P(x)$.

\subsection{Finite-Time Stabilization of Continuous-Time Polynomial} Fuzzy System. In this subsection, a finite-time fuzzy controller with polynomial rule consequence is designed for the system (3) and the control rule shares the same IF parts with system (3) as follows.

Control Rule i. If $z_{1}(t)$ is $\mathscr{M}_{i 1}$ and $z_{2}(t)$ is $\mathscr{M}_{i 2}$ and $z_{p}(t)$ is $M_{i p}$, then

$$
u(t)=-F_{i}(x(t)) \hat{x}(x(t)), \quad i=1,2, \ldots, r .
$$

Referring to [10], the overall fuzzy controller can be calculated by

$$
u(t)=-\sum_{i=1}^{r} h_{i}(z(t)) F_{i}(x(t)) \widehat{x}(x(t))
$$

and the closed-loop system (3) can be rewritten as

$$
\begin{aligned}
\dot{x}(t) & =\sum_{i=1}^{r} \sum_{j=1}^{r} h_{i}(z(t)) h_{j}(z(t)) \\
\cdot & \left\{A_{i}(x(t))-B_{i}(x(t)) F_{j}(x(t))\right\} \hat{x}(x(t)) .
\end{aligned}
$$

From now on, to lighten the notation, we will employ the same method as what has been used in Section 3.1. In addition, let $K=\left\{k_{1}, k_{2}, \ldots, k_{m}\right\}$ denote the row indices of $B_{i}(x)$ whose corresponding row is equal to zero, and define $\tilde{x}=\left(x_{k_{1}}, x_{k_{2}}, \ldots, x_{k_{m}}\right)$.

Theorem 10. Suppose there exist a symmetric polynomial matrix $X(\tilde{x}) \in R^{N \times N}$, a polynomial matrix $M_{i}(x) \in R^{m \times N}$, a constant $\alpha \geq 0$, and two positive scalars $\Phi_{1}$ and $\Phi_{2}$ such that the following (29)-(33) are satisfied:

$$
\begin{aligned}
& \eta_{1}^{T}\left(X(\tilde{x})-\epsilon_{1}(x) I\right) \eta_{1} \text { is SOS, } \\
& -\eta_{1}^{T}\left(T(x) A_{i}(x) X(\tilde{x})-T(x) B_{i}(x) M_{j}(x)\right. \\
& \quad+X(\tilde{x}) A_{i}^{T}(x) T^{T}(x)-M_{j}^{T}(x) B_{i}^{T}(x) T^{T}(x) \\
& \quad+T(x) A_{j}(x) X(\tilde{x})-T(x) B_{j}(x) M_{i}(x) \\
& \quad+X(\tilde{x}) A_{j}^{T}(x) T^{T}(x)-M_{i}^{T}(x) B_{j}^{T}(x) T^{T}(x) \\
& \quad-\sum_{k \in K} \frac{\partial X(\tilde{x})}{\partial x_{k}} A_{i}^{k}(x) \widehat{x}(x)-\sum_{k \in K} \frac{\partial X(\tilde{x})}{\partial x_{k}} A_{j}^{k}(x) \widehat{x}(x) \\
& \left.-2 \alpha X(\tilde{x})+\epsilon_{2 i j}(x) I\right) \eta_{1} \text { is } S O S, \quad i \leq j,
\end{aligned}
$$




$$
\begin{aligned}
& \eta_{1}^{T}\left(X(\tilde{x}) R-\Phi_{1} I-\epsilon_{2}(x) I\right) \eta_{1} \text { is SOS, } \\
& \eta_{1}^{T}\left(\Phi_{2} I-X(\tilde{x}) R-\epsilon_{3}(x) I\right) \eta_{1} \text { is SOS, } \\
& \eta_{2}^{T}\left(\Phi_{1} c_{2}-\Phi_{2} c_{1} e^{\alpha T_{f}}-\epsilon_{4}(x)\right) \eta_{2} \text { is SOS, }
\end{aligned}
$$

where $T(x)$ is the same as (13); polynomials $\epsilon_{1}(x)>0, \epsilon_{2}(x)>$ $0, \epsilon_{2 i j}(x)>0, \epsilon_{3}(x)>0, \epsilon_{4}(x)>0$ for $x \neq 0 ; \eta_{1} \in R^{N}$, $\eta_{2} \in R$ are two vectors independent of $x$; then the continuoustime polynomial fuzzy system (28) is finite-time stable with respect to $\left(c_{1}, c_{2}, T_{f}, R\right)$. That is to say, system (3) is finite-time stabilization with respect to $\left(c_{1}, c_{2}, T_{f}, R\right)$ with the feedback gain

$$
F_{i}(x)=M_{i}(x) X^{-1}(\tilde{x}) .
$$

Proof. Choose the following Lyapunov functional:

$$
V(x)=\widehat{x}^{T}(x) X^{-1}(\tilde{x}) \widehat{x}(x),
$$

where $X^{-1}(\tilde{x}) \in R^{N \times N}$ is a symmetric polynomial matrix. From condition (29), which implies that both $X(\tilde{x})$ and $X^{-1}(\tilde{x})$ are positive definite for all $x$, we can obtain that $V(x)$ is a positive definite function of $x$.

Considering $B_{i}^{k}(x)=0$ for $k \in K$ and $\left(\partial X^{-1} / \partial x_{i}\right)(\tilde{x})=0$ for $i \notin K$, the time derivative of $V(x)$ along (28) is given by

$$
\begin{aligned}
& \dot{V}(x)=\widehat{x}^{T}(x) X^{-1}(\widetilde{x}) \dot{\hat{x}}(x)+\dot{\hat{x}}^{T}(x) X^{-1}(\widetilde{x}) \hat{x}(x) \\
& +\widehat{x}^{T}(x) \dot{X}^{-1}(\tilde{x}) \hat{x}(x)=\widehat{x}^{T}(x) X^{-1}(\tilde{x}) T(x) \dot{x}(x) \\
& +\dot{x}^{T}(x) T^{T}(x) X^{-1}(\tilde{x}) \hat{x}(x)+\widehat{x}^{T}(x)\left(\sum_{k=1}^{n} \frac{\partial X^{-1}}{\partial x_{k}}\right. \\
& \left.\cdot(\tilde{x}) \dot{x}_{k}\right) \widehat{x}(x)=\widehat{x}^{T}(x) X^{-1}(\tilde{x}) T(x) \dot{x}(x) \\
& +\dot{x}^{T}(x) T^{T}(x) X^{-1}(\tilde{x}) \hat{x}(x)+\widehat{x}^{T}(x)\left(\sum_{k=1}^{n} \frac{\partial X^{-1}}{\partial x_{k}}\right. \\
& \left.\cdot(\tilde{x}) \sum_{i=1}^{r} h_{i}(z) A_{i}^{k}(x) \hat{x}(x)\right) \widehat{x}(x)=\sum_{i=1}^{r} \sum_{j=1}^{r} h_{i}(z) \\
& \cdot h_{j}(z) \hat{x}^{T}(x) \\
& \cdot\left(\Omega_{i j}(x)+\sum_{k \in K} \frac{\partial X^{-1}}{\partial x_{k}}(\tilde{x}) A_{i}^{k}(x) \widehat{x}(x)\right) \widehat{x}(x)=\frac{1}{2} \\
& \cdot \sum_{i=1}^{r} \sum_{j=1}^{r} h_{i}(z) h_{j}(z) \hat{x}^{T}(x)\left(\Omega_{i j}(x)+\Omega_{j i}(x)\right. \\
& +\sum_{k \in K} \frac{\partial X^{-1}}{\partial x_{k}}(\widetilde{x}) A_{i}^{k}(x) \widehat{x}(x)+\sum_{k \in K} \frac{\partial X^{-1}}{\partial x_{k}}(\widetilde{x}) A_{j}^{k}(x) \\
& \widehat{x}(x)) \widehat{x}(x)
\end{aligned}
$$

where $\Omega_{i j}(x)=X^{-1}(\tilde{x}) T(x)\left\{A_{i}(x)-B_{i}(x) F_{j}(x)\right\}+\left\{A_{i}(x)-\right.$ $\left.B_{i}(x) F_{j}(x)\right\}^{T} T^{T}(x) X^{-1}(\tilde{x})$. According to Lemma 6 , condition (30) implies that

$$
\begin{aligned}
& -\left(T(x) A_{i}(x) X(\tilde{x})-T(x) B_{i}(x) M_{j}(x)\right. \\
& +X(\tilde{x}) A_{i}^{T}(x) T^{T}(x)-M_{j}^{T}(x) B_{i}^{T}(x) T^{T}(x) \\
& +T(x) A_{j}(x) X(\tilde{x})-T(x) B_{j}(x) M_{i}(x) \\
& +X(\tilde{x}) A_{j}^{T}(x) T^{T}(x)-M_{i}^{T}(x) B_{j}^{T}(x) T^{T}(x) \\
& -\sum_{k \in K} \frac{\partial X(\tilde{x})}{\partial x_{k}} A_{i}^{k}(x) \hat{x}(x)-\sum_{k \in K} \frac{\partial X(\tilde{x})}{\partial x_{k}} A_{j}^{k}(x) \hat{x}(x) \\
& -2 \alpha X(\tilde{x})) \geq 0,
\end{aligned}
$$

for $i \leq j$ and all $x$. Taking (34) into consideration and multiplying (37) from the left and right by $X^{-1}(\tilde{x})$, we have

$$
\begin{aligned}
& -\left\{\Omega_{i j}(x)+\Omega_{j i}(x)-2 \alpha X^{-1}(\tilde{x})\right. \\
& -\sum_{k \in K} X^{-1}(\tilde{x}) \frac{\partial X}{\partial x_{k}}(\tilde{x}) X^{-1}(\tilde{x}) A_{i}^{k}(x) \widehat{x}(x) \\
& \left.-\sum_{k \in K} X^{-1}(\tilde{x}) \frac{\partial X}{\partial x_{k}}(\tilde{x}) X^{-1}(\tilde{x}) A_{j}^{k}(x) \widehat{x}(x)\right\} \geq 0 .
\end{aligned}
$$

It is from differentiating both sides of the relation $X^{-1}(\tilde{x}) X(\tilde{x})=I$ with respect to $x_{k}$ that

$$
X^{-1}(\tilde{x}) \frac{\partial X}{\partial x_{k}}(\tilde{x}) X^{-1}(\tilde{x})=-\frac{\partial X^{-1}}{\partial x_{k}}(\tilde{x}) .
$$

Therefore, it is from (30), (38), and (39) that

$$
\begin{aligned}
\Omega_{i j}(x) & +\Omega_{j i}(x)+\sum_{k \in K} \frac{\partial X^{-1}}{\partial x_{k}}(\tilde{x}) A_{i}^{k}(x) \hat{x}(x) \\
& +\sum_{k \in K} \frac{\partial X^{-1}}{\partial x_{k}}(\tilde{x}) A_{j}^{k}(x) \hat{x}(x)<2 \alpha X^{-1}(\tilde{x}),
\end{aligned}
$$

at $x \neq 0$.

Putting together (36) and (40), we can obtain

$$
\begin{aligned}
\dot{V}(x) & <\sum_{i=1}^{r} \sum_{j=1}^{r} h_{i}(z) h_{j}(z) \widehat{x}^{T}(x)\left\{\alpha X^{-1}(\tilde{x})\right\} \widehat{x}(x) \\
& =\alpha \widehat{x}^{T}(x) X^{-1}(\tilde{x}) \hat{x}(x)=\alpha V(x) .
\end{aligned}
$$

Integrating (41) from 0 to $t, t \in\left(0, T_{f}\right]$, we have

$$
V(x)<e^{\alpha t} V(x(0)) .
$$


It is from (31) and (32) that

$$
\begin{aligned}
\Phi_{1} I & \leq X(\tilde{x}) R \\
& \Longrightarrow X^{-1}(\tilde{x}) \Phi_{1} I \leq R \\
& \Longrightarrow X^{-1}(\tilde{x}) \leq R\left(\Phi_{1} I\right)^{-1} \\
& \Longrightarrow X^{-1}(\tilde{x}) \leq \frac{R}{\Phi_{1}}, \\
\Phi_{2} I & \geq X(\tilde{x}) R \\
& \Longrightarrow X^{-1}(\tilde{x}) \Phi_{2} I \geq R \\
& \Longrightarrow X^{-1}(\tilde{x}) \geq R\left(\Phi_{2} I\right)^{-1} \\
& \Longrightarrow X^{-1}(\tilde{x}) \geq \frac{R}{\Phi_{2}} .
\end{aligned}
$$

Then, we can obtain the following:

$$
\begin{aligned}
V(x) & =\widehat{x}^{T}(x) X^{-1}(\tilde{x}) \widehat{x}(x) \geq \widehat{x}^{T}(x) \frac{R}{\Phi_{2}} \widehat{x}(x) \\
& =\frac{1}{\Phi_{2}} \widehat{x}^{T}(x) R \widehat{x}(x), \\
V(x(0)) & =\widehat{x}^{T}(x(0)) X^{-1}(\tilde{x}) \hat{x}(x(0)) \\
& \leq \widehat{x}^{T}(x(0)) \frac{R}{\Phi_{1}} \widehat{x}(x(0)) \\
& =\frac{1}{\Phi_{1}} \widehat{x}^{T}(x(0)) R \widehat{x}(x(0)) .
\end{aligned}
$$

Considering $\widehat{x}^{T}(x(0)) R \widehat{x}(x(0))<c_{1}$ and (42)-(44), we get

$$
\begin{aligned}
\hat{x}^{T}(x) R \widehat{x}(x) & \leq \Phi_{2} V(x)<\Phi_{2} e^{\alpha t} V(x(0)) \\
& \leq \Phi_{2} e^{\alpha T_{f}} V(x(0)) \\
& \leq \Phi_{2} e^{\alpha T_{f}} \frac{1}{\Phi_{1}} \hat{x}^{T}(x(0)) R \hat{x}(x(0)) \\
& <\frac{\Phi_{2}}{\Phi_{1}} c_{1} e^{\alpha T_{f}} .
\end{aligned}
$$

Assume that condition (33) is satisfied, which means $\Phi_{2} c_{1} e^{\alpha T_{f}} \leq \Phi_{1} c_{2}$, then

$$
\hat{x}^{T}(x(t)) R \hat{x}(x(t))<\frac{\Phi_{2} c_{1} e^{\alpha T_{f}}}{\Phi_{1}} \leq \frac{\Phi_{1} c_{2}}{\Phi_{1}}=c_{2} .
$$

According to Definition 4, system (28) is finite-time stabilization with respect to $\left(c_{1}, c_{2}, T_{f}, R\right)$. That is to say, the continuous-time polynomial fuzzy system (3) is finite-time stabilization with respect to $\left(c_{1}, c_{2}, T_{f}, R\right)$ with the fuzzy controlleres (26) and (34). This completes the proof.

Remark 11. When $A_{i}(x), M_{i}(x)$, and $X(\tilde{x})$ in Theorem 10 reduce to constant matrices $A, M$, and $X$, the finite-time stabilization conditions of the continuous-time polynomial fuzzy system (3) reduce to the one in Theorem 5 of [24], which means that the classical result is a special case of ours.

\section{Numerical Simulations}

To illustrate the effectiveness and validity of our main results, this section provides three illustrative examples.

Example 1. Consider the following nonlinear system:

$$
\begin{aligned}
& \dot{x}_{1}=-4.5 x_{1}-0.5 x_{1}^{2}-4 x_{2}, \\
& \dot{x}_{2}=9.5 x_{1}-10.5 \sin x_{1}-2 x_{2} .
\end{aligned}
$$

Using the sector nonlinearity technique [1], the nonlinear system (47) is exactly converted into the following two-rule polynomial fuzzy system:

$$
\dot{x}=\sum_{i=1}^{2} h_{i}(z)\left\{A_{i}(x) \widehat{x}(x)\right\},
$$

where $x=\widehat{x}(x)=\left[\begin{array}{ll}x_{1} & x_{2}\end{array}\right]^{T}, z=x_{1}$, and

$$
\begin{aligned}
& A_{1}(x)=\left[\begin{array}{cc}
-4.5-0.5 x_{1} & -4 \\
-1 & -2
\end{array}\right], \\
& A_{2}(x)=\left[\begin{array}{cc}
-4.5-0.5 x_{1} & -4 \\
11.7806 & -2
\end{array}\right], \\
& h_{1}(z)=\frac{\sin x_{1}+0.2172 x_{1}}{1.2172 x_{1}}, \\
& h_{2}(z)=\frac{x_{1}-\sin x_{1}}{1.2172 x_{1}} .
\end{aligned}
$$

The corresponding parameters for the finite-time stability are given in this example as follows:

$$
\begin{aligned}
c_{1} & =1, \\
c_{2} & =45, \\
T_{f} & =5, \\
\alpha & =1.5, \\
R & =I .
\end{aligned}
$$

By using the SOSTOOLS of Matlab and solving Theorem 7 , we can get the following feasible $P(x)$, and corresponding $\Phi_{1}$, $\Phi_{2}$ for system (47):

$$
\begin{aligned}
P(x) & =\left[\begin{array}{cc}
1.816 e^{-8} & 4.922 e^{-11} \\
4.922 e^{-11} & 1.448 e^{-8}
\end{array}\right], \\
\Phi_{1} & =1.163 e^{-8}, \\
\Phi_{2} & =0.7978,
\end{aligned}
$$

where the degree of the polynomial $P(x)$ is set to be 0 . It is obvious that when the degree of $P(x)$ is set different from 0 , different $\Phi_{i}, i=1,2$, will be obtained accordingly. Notation. In this section, $e^{-N}$ stands for $10^{-N}$. 


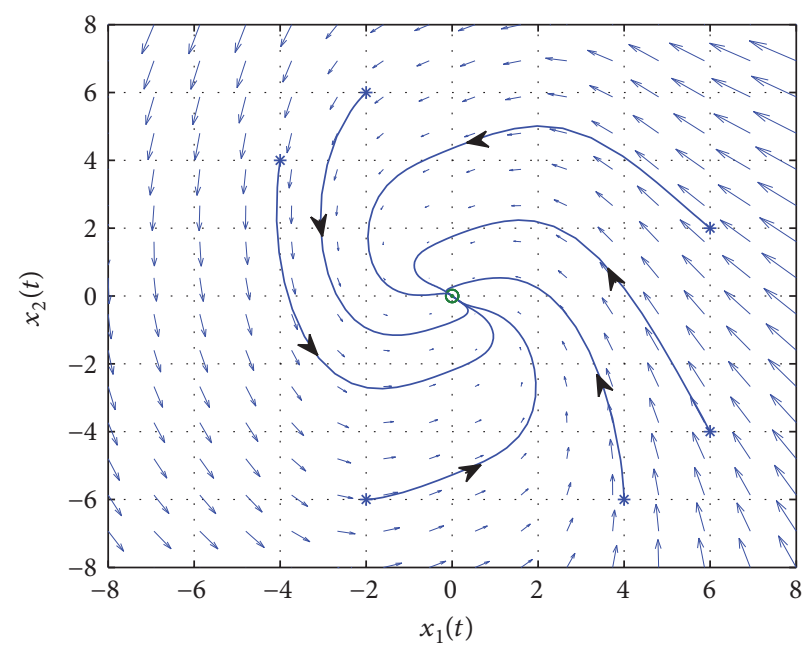

Figure 1: Behaviors of nonlinear system (47) in $x_{1}-x_{2}$ plane.
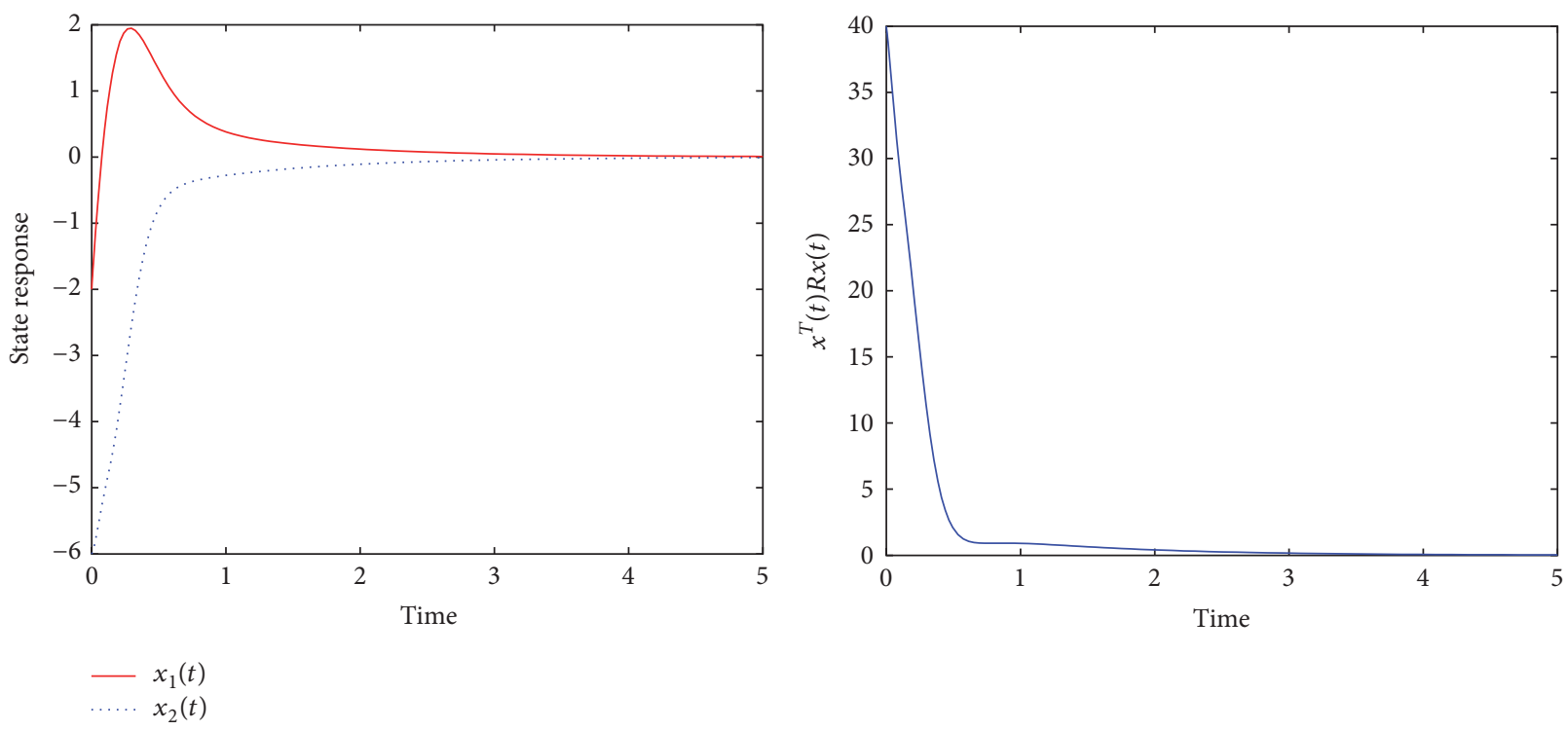

FIGURE 2: The state responses of polynomial fuzzy system (47) for the initial point $x(0)=\left[\begin{array}{ll}-2-6 & -6\end{array}\right]^{T}$.

Figure 1 shows the phase graph of system (47) for six different initial points $(-2,6),(-4,4),(-2,-6),(4,-6)$, $(6,-4)$, and $(6,2)$ and Figure 2 shows its state response for $x(0)=(-2,-6)$, from which it is easy to see that system (47) is finite-time stable.

Example 2. Consider the following nonlinear system with control input $u$ :

$$
\begin{aligned}
& \dot{x}_{1}=-3 x_{1}+2 x_{1}^{3}+2 x_{1}^{2} x_{2}-3 x_{1} x_{2}^{2}+7 x_{2}+x_{1} u, \\
& \dot{x}_{2}=-2 \sin x_{1}-3 x_{2} .
\end{aligned}
$$

Figure 3(a) shows the behaviors of system (52) with $u=0$ for six different initial points $(4,6),(-2,6),(-4,4),(-2,-6)$, $(4,-6)$, and $(6,-4)$. It is easy to see that system (52) without control input is unstable.
Using the same technique as before, the nonlinear system (52) can be exactly converted into the following two-rule polynomial fuzzy system:

$$
\dot{x}=\sum_{i=1}^{2} h_{i}(z)\left\{A_{i}(x) \widehat{x}(x)+B_{i}(x) u\right\},
$$

where $x=\widehat{x}(x)=\left[\begin{array}{ll}x_{1} & x_{2}\end{array}\right]^{T}, z=x_{1}$, and

$$
\begin{aligned}
& A_{1}(x)=\left[\begin{array}{cc}
-3+2 x_{1}^{2}+2 x_{1} x_{2}-3 x_{2}^{2} & 7 \\
-2 & -3
\end{array}\right], \\
& A_{2}(x)=\left[\begin{array}{cc}
-3+2 x_{1}^{2}+2 x_{1} x_{2}-3 x_{2}^{2} & 7 \\
0.4344 & -3
\end{array}\right], \\
& B_{1}(x)=\left[\begin{array}{c}
x_{1} \\
0
\end{array}\right],
\end{aligned}
$$




$$
\begin{aligned}
& B_{2}(x)=\left[\begin{array}{l}
x_{1} \\
0
\end{array}\right], \\
& h_{1}(z)=\frac{\sin x_{1}+0.2172 x_{1}}{1.2172 x_{1}}, \\
& h_{2}(z)=\frac{x_{1}-\sin x_{1}}{1.2172 x_{1}} .
\end{aligned}
$$

The corresponding parameters for the finite-time stabilization are given in this example as follows:

$$
\begin{aligned}
& c_{1}=1, \\
& c_{2}=45,
\end{aligned}
$$

$$
\begin{aligned}
& F_{1}(x)=\left[\begin{array}{ll}
3.89 x_{1}+1.822 x_{2}-2.904 e^{-8} & 8.757 e^{-6} x_{1}-4.138 e^{-7} x_{2}-3.946 e^{-8}
\end{array}\right], \\
& F_{2}(x)=\left[\begin{array}{ll}
3.89 x_{1}+1.822 x_{2}-6.042 e^{-8} & 8.757 e^{-6} x_{1}-4.138 e^{-7} x_{2}+2.36 e^{-7}
\end{array}\right],
\end{aligned}
$$

respectively, where the degree of the polynomial $M_{i}(x)$ is set to be 0 to 1 (code for SOSTOOLS). When the degree is set to be different from what is set here, different $F_{i}(x)$ will be obtained accordingly.

Figures 3(b) and 4 show the validity and effectiveness of our proposed method.

Example 3. As shown in [31], a coaxial counter rotating helicopter dynamics can be written as

$$
\begin{aligned}
& \dot{u}=-\frac{a}{I_{z}} \Psi v+K U_{X}, \\
& \dot{v}=\frac{a}{I_{z}} \Psi u+K U_{Y}, \\
& \dot{w}=K U_{Z},
\end{aligned}
$$

where $a=1.5, I_{z}=0.2857$, and the other corresponding variables such as $u, v, w, \Psi, U_{X}, U_{Y}$, and $U_{Z}$ are the same as [31]. For the lack of space, the details are omitted here.

As above, nonlinear system (59) can be exactly converted into the following two-rule polynomial fuzzy system for $\Psi \in$ $\left[\begin{array}{ll}-\pi & \pi\end{array}\right]$ :

$$
\dot{x}=\sum_{i=1}^{2} h_{i}(z)\left\{A_{i}(x) \widehat{x}(x)+B_{i}(x) u\right\}
$$

where $x=\widehat{x}(x)=\left[\begin{array}{llllll}u & v & w & e_{x} & e_{y} & e_{z}\end{array}\right]^{T}, z=\Psi$, and $u=\left[\begin{array}{lll}U_{X} & U_{Y} & U_{Z}\end{array}\right]^{T} . e_{x}, e_{y}, e_{z}$, and $A_{i}(x)$ matrices are defined the same as in [31]. Besides, $B_{i}(x)$ and the membership functions are given as follows:

$$
\begin{aligned}
& B_{1}(x)=B_{2}(x)=\left[\begin{array}{lll}
K & 0 & 0 \\
0 & K & 0 \\
0 & 0 & K \\
0 & 0 & 0 \\
0 & 0 & 0 \\
0 & 0 & 0
\end{array}\right], \\
& h_{1}(z)=\frac{z+\pi}{2 \pi}, \\
& h_{2}(z)=\frac{\pi-z}{2 \pi} .
\end{aligned}
$$

and the feedback gains $F_{1}(x)$ and $F_{2}(x)$ are obtained as follows:

The corresponding parameters for the finite-time stabilization are given in this example as follows:

$$
\begin{aligned}
K & =5, \\
c_{1} & =1, \\
c_{2} & =5, \\
T_{f} & =10, \\
\alpha & =1.5, \\
R & =I .
\end{aligned}
$$

By using the SOSTOOLS of Matlab and solving Theorem 10, the solutions are found as follows: 


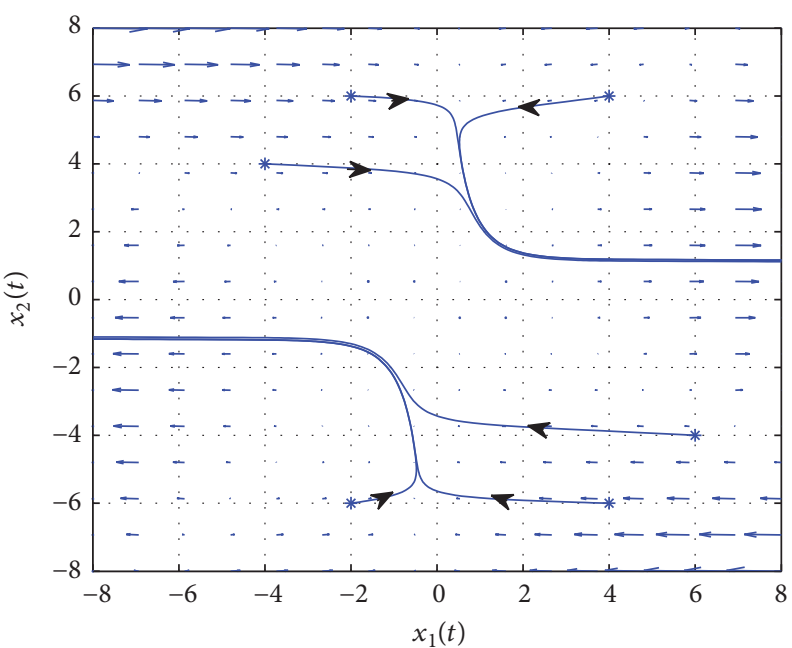

(a)

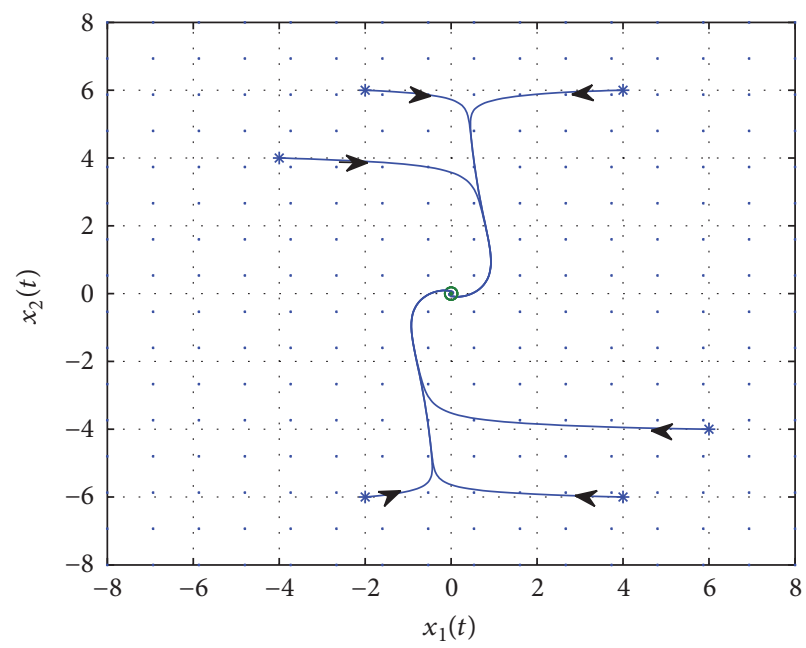

(b)

FIGURE 3: Behaviors of nonlinear system (52) in $x_{1}-x_{2}$ plane. (a) depicts the movement trajectories of system (52) with $u=0$ for the initial points of $(4,6),(-2,6),(-4,4),(-2,-6),(4,-6)$, and $(6,-4)$ and corresponding movement directions represented by arrows; (b) shows the behaviors of system (52) with control input for the same initial points as (a).
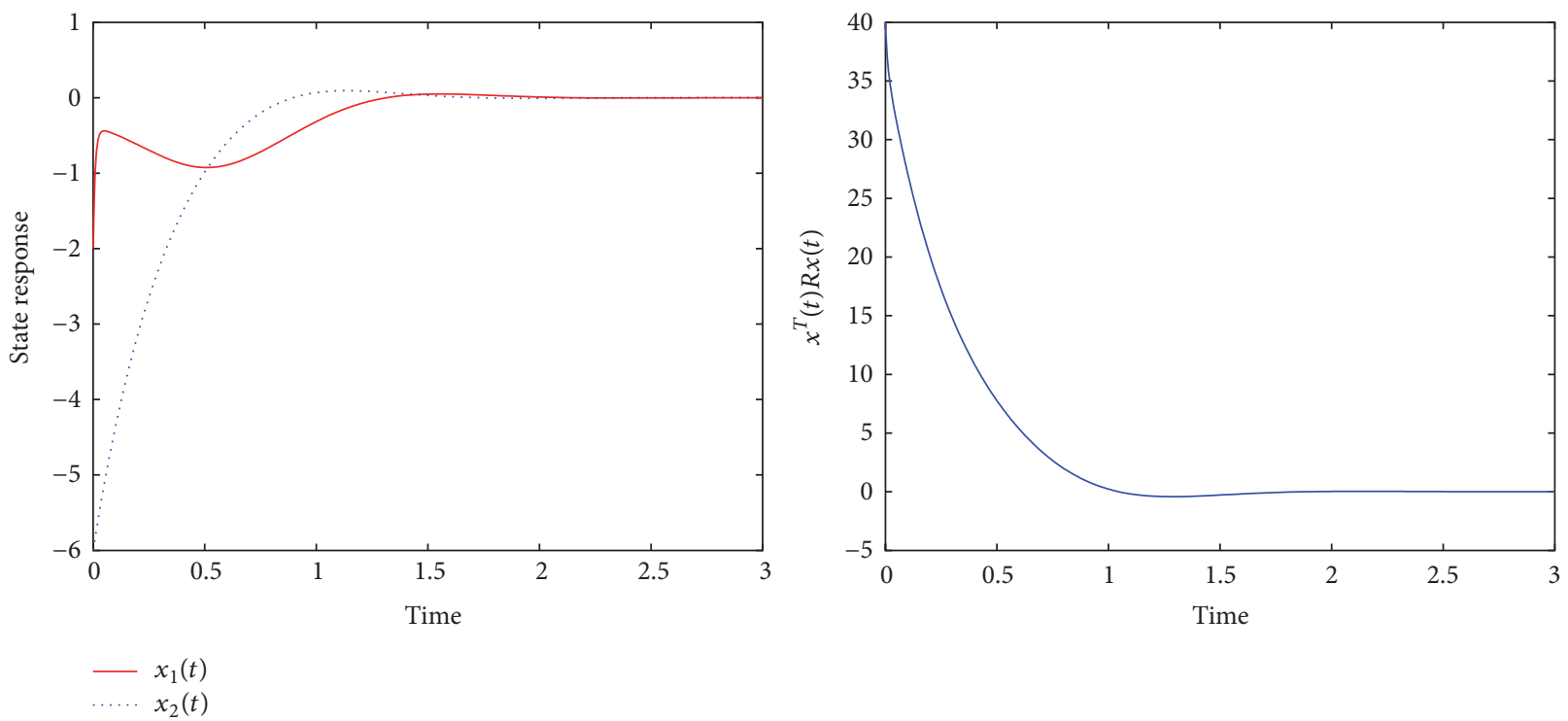

FIGURE 4: The state responses of polynomial fuzzy system (52) for the initial point $x(0)=\left[\begin{array}{ll}-2 & -6\end{array}\right]^{T}$.

$$
\begin{aligned}
X(\tilde{x}) & =\left[\begin{array}{cccccc}
0.986 & 1.555 e^{-9} & -3.702 e^{-10} & 0.1232 & 1.07 e^{-8} & 9.019 e^{-9} \\
1.555 e^{-9} & 0.986 & 7.595 e^{-9} & 1.069 e^{-9} & 0.1232 & 1.147 e^{-8} \\
-3.702 e^{-10} & 7.595 e^{-9} & 0.9992 & 4.862 e^{-9} & 3.671 e^{-9} & 0.1854 \\
0.1232 & 1.069 e^{-9} & 4.862 e^{-9} & 0.682 & -1.559 e^{-8} & -5.253 e^{-9} \\
1.07 e^{-8} & 0.1232 & 3.671 e^{-9} & -1.559 e^{-8} & 0.682 & -8.217 e^{-9} \\
9.019 e^{-9} & 1.147 e^{-8} & 0.1854 & -5.253 e^{-9} & -8.217 e^{-9} & 0.7408
\end{array}\right], \\
\Phi_{1} & =0.1258 \\
\Phi_{2} & =1.945
\end{aligned}
$$


and the feedback gains $F_{1}(x)$ and $F_{2}(x)$ are obtained as follows:

$$
\begin{aligned}
& F_{1}(x) \\
& =\left[\begin{array}{llllll}
F_{111}(x) & F_{112}(x) & F_{113}(x) & F_{114}(x) & F_{115}(x) & F_{116}(x) \\
F_{121}(x) & F_{122}(x) & F_{123}(x) & F_{124}(x) & F_{125}(x) & F_{126}(x) \\
F_{131}(x) & F_{132}(x) & F_{133}(x) & F_{134}(x) & F_{135}(x) & F_{136}(x)
\end{array}\right],
\end{aligned}
$$

$F_{2}(x)$

$$
=\left[\begin{array}{llllll}
F_{211}(x) & F_{212}(x) & F_{213}(x) & F_{214}(x) & F_{215}(x) & F_{216}(x) \\
F_{221}(x) & F_{222}(x) & F_{223}(x) & F_{224}(x) & F_{225}(x) & F_{226}(x) \\
F_{231}(x) & F_{232}(x) & F_{233}(x) & F_{234}(x) & F_{235}(x) & F_{236}(x)
\end{array}\right],
$$

respectively, where $F_{111}(x)=-6.099 e^{-19} u-2.148 e^{-19} v+$ $9.783 e^{-19} w+3.805 e^{-18} e_{x}-1.239 e^{-18} e_{y}-8.226 e^{-19} e_{z}-$ $0.1191, F_{112}(x)=-1.354 e^{-18} u-7.619 e^{-19} v-4.773 e^{-18} w-$ $3.675 e^{-18} e_{x}-1.029 e^{-17} e_{y}+2.019 e^{-18} e_{z}+0.07589, F_{113}(x)=$ $-2.0 e^{-19} u+6.485 e^{-18} v-7.584 e^{-18} w-1.004 e^{-19} e_{x}+5.0 e^{-18} e_{y}-$ $2.339 e^{-18} e_{z}-1.291 e^{-9}, F_{114}(x)=4.969 e^{-18} u+1.693 e^{-18} v-$ $8.105 e^{-18} w-2.866 e^{-17} e_{x}+9.703 e^{-18} e_{y}+6.396 e^{-18} e_{z}+0.2559$, $F_{115}(x)=1.162 e^{-17} u+4.136 e^{-18} v+8.305 e^{-19} w+4.104 e^{-17} e_{x}-$ $3.547 e^{-18} e_{y}-1.524 e^{-17} e_{z}-0.6076, F_{116}(x)=1.043 e^{-18} u-$ $3.551 e^{-17} v+8.546 e^{-19} w+1.871 e^{-18} e_{x}+5.035 e^{-18} e_{y}+$ $2.118 e^{-18} e_{z}-5.846 e^{-8}, F_{121}(x)=-6.86 e^{-19} u+8.991 e^{-19} v-$ $1.918 e^{-18} w+2.11 e^{-18} e_{x}-1.082 e^{-17} e_{y}+4.249 e^{-18} e_{z}-0.07589$, $F_{122}(x)=3.94 e^{-18} u+1.244 e^{-17} v+4.496 e^{-18} w+8.611 e^{-18} e_{x}+$ $8.183 e^{-18} e_{y}+4.86 e^{-18} e_{z}-0.1191, F_{123}(x)=-1.051 e^{-18} u+$ $5.089 e^{-18} v-2.835 e^{-18} w+4.915 e^{-18} e_{x}+2.679 e^{-18} e_{y}+$ $3.857 e^{-18} e_{z}-1.389 e^{-8}, F_{124}(x)=6.27 e^{-18} u-9.161 e^{-18} v-$ $2.203 e^{-17} w-5.276 e^{-18} e_{x}+6.848 e^{-19} e_{y}-3.309 e^{-17} e_{z}+0.6076$, $F_{125}(x)=1.036 e^{-18} u-8.734 e^{-19} v-5.028 e^{-20} w+4.953 e^{-18} e_{x}-$ $2.698 e^{-17} e_{y}-3.998 e^{-17} e_{z}+0.2559, F_{126}(x)=3.609 e^{-18} u-$ $1.008 e^{-17} v+8.839 e^{-18} w+5.553 e^{-19} e_{x}-4.033 e^{-18} e_{y}-$ $5.843 e^{-18} e_{z}+5.096 e^{-8}, F_{131}(x)=1.481 e^{-18} u+1.764 e^{-18} v-$ $6.393 e^{-18} w-2.935 e^{-18} e_{x}+5.046 e^{-18} e_{y}-1.529 e^{-18} e_{z}-$ $1.526 e^{-8}, F_{132}(x)=-4.78 e^{-19} u-2.696 e^{-19} v+2.256 e^{-18} w+$ $5.715 e^{-18} e_{x}-2.675 e^{-18} e_{y}-1.446 e^{-18} e_{z}-3.809 e^{-9}, F_{133}(x)=$ $-6.882 e^{-19} u-7.187 e^{-18} v+4.277 e^{-19} w-1.682 e^{-18} e_{x}-$ $5.33 e^{-18} e_{y}-1.446 e^{-18} e_{z}-0.13, F_{134}(x)=-1.191 e^{-17} u-$ $1.496 e^{-17} v-9.053 e^{-18} w+2.55 e^{-17} e_{x}+7.743 e^{-18} e_{y}-$ $3.548 e^{-18} e_{z}+3.701 e^{-9}, F_{135}(x)=7.295 e^{-19} u+2.827 e^{-17} v-$ $2.776 e^{-17} w-5.046 e^{-18} e_{x}+3.708 e^{-17} e_{y}+3.407 e^{-17} e_{z}+5.927 e^{-9}$, $F_{136}(x)=1.037 e^{-18} u+1.434 e^{-18} v-1.866 e^{-18} w+4.03 e^{-18} e_{x}+$ $5.185 e^{-18} e_{y}-7.855 e^{-18} e_{z}+0.2267, F_{211}(x)=3.725 e^{-18} u-$ $9.806 e^{-20} v+8.645 e^{-19} w+3.864 e^{-18} e_{x}-1.32 e^{-18} e_{y}+$ $3.901 e^{-18} e_{z}-0.1191, F_{212}(x)=-2.925 e^{-18} u+2.344 e^{-19} v-$ $4.923 e^{-18} w+1.217 e^{-18} e_{x}-5.644 e^{-19} e_{y}+1.843 e^{-18} e_{z}-$ $0.07589, F_{213}(x)=3.162 e^{-20} u+7.686 e^{-18} v-7.392 e^{-18} w-$ $3.734 e^{-19} e_{x}+4.376 e^{-18} e_{y}-2.143 e^{-18} e_{z}-1.348 e^{-9}, F_{214}(x)=$ $-3.086 e^{-17} u-1.348 e^{-18} v-5.816 e^{-18} w-2.903 e^{-17} e_{x}+$ $1.071 e^{-17} e_{y}-3.158 e^{-17} e_{z}+0.2559, F_{215}(x)=1.977 e^{-17} u-$ $1.903 e^{-18} v+3.117 e^{-18} w-2.782 e^{-17} e_{x}+5.987 e^{-18} e_{y}-$ $1.429 e^{-17} e_{z}+0.6076, F_{216}(x)=-1.864 e^{-19} u-4.124 e^{-17} v+$

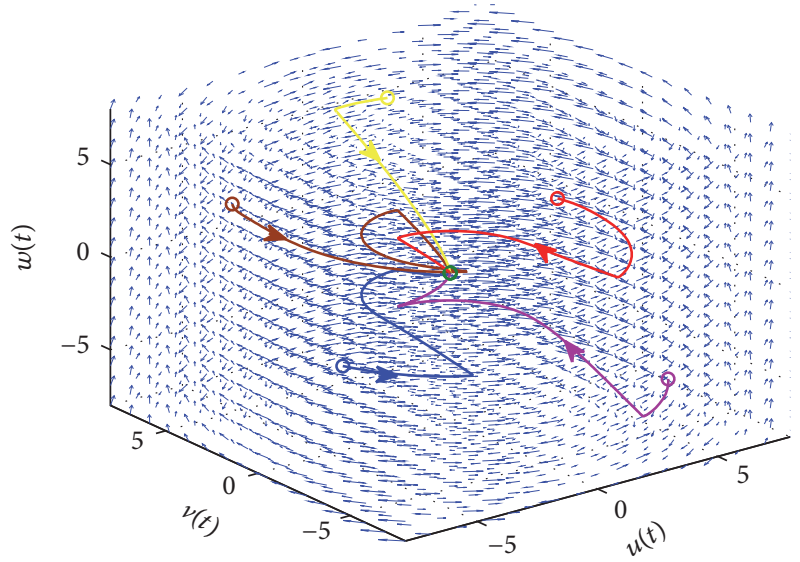

FIGURE 5: Behaviors of the coaxial counter rotating helicopter dynamical system (59) in $u(t)-v(t)-w(t)$ plane, which shows the behaviors of system (59) with control input for the initial points of $(-6,-2,-2),(-6,4,4),(2,6,6),(6,-4,-6)$, and $(6,2,1)$.

$8.697 e^{-19} w+2.472 e^{-18} e_{x}+6.632 e^{-18} e_{y}+7.074 e^{-19} e_{z}+5.92 e^{-8}$, $F_{221}(x)=2.599 e^{-19} u+9.73 e^{-19} v-4.299 e^{-18} w-3.93 e^{-18} e_{x}+$ $1.301 e^{-18} e_{y}+7.589 e^{-19} e_{z}+0.07589, F_{222}(x)=6.73 e^{-20} u-$ $2.495 e^{-18} v-2.773 e^{-18} w-2.285 e^{-19} e_{x}-2.825 e^{-19} e_{y}-$ $1.943 e^{-18} e_{z}-0.1191, F_{223}(x)=-4.836 e^{-19} u+2.026 e^{-18} v-$ $3.325 e^{-18} w+4.534 e^{-18} e_{x}+3.383 e^{-18} e_{y}+1.603 e^{-18} e_{z}+1.126 e^{-8}$, $F_{224}(x)=-5.728 e^{-18} u-7.816 e^{-18} v-1.876 e^{-18} w+1.34 e^{-17} e_{x}$ $8.949 e^{-18} e_{y}-5.611 e^{-18} e_{z}-0.6076, F_{225}(x)=-1.185 e^{-18} u+$ $2.124 e^{-18} v+3.844 e^{-18} w-1.471 e^{-17} e_{x}-9.184 e^{-18} e_{y}+$ $1.134 e^{-17} e_{z}+0.2559, F_{226}(x)=-3.293 e^{-19} u-4.59 e^{-18} v+$ $9.91 e^{-18} w+1.976 e^{-18} e_{x}-6.461 e^{-18} e_{y}+1.188 e^{-19} e_{z}-4.571 e^{-8}$, $F_{231}(x)=-4.934 e^{-19} u-1.488 e^{-19} v-4.76 e^{-18} w-1.532 e^{-18} e_{x}+$ $2.569 e^{-18} e_{y}-3.903 e^{-18} e_{z}+1.073 e^{-8}, F_{232}(x)=-1.319 e^{-18} u+$ $1.944 e^{-18} v+2.393 e^{-18} w+4.599 e^{-18} e_{x}-1.246 e^{-18} e_{y}+$ $4.66 e^{-20} e_{z}-2.479 e^{-9}, F_{233}(x)=5.347 e^{-19} u+3.65 e^{-17} v+$ $3.174 e^{-18} w-3.972 e^{-19} e_{x}-3.877 e^{-18} e_{y}-3.724 e^{-18} e_{z}-0.13$, $F_{234}(x)=3.926 e^{-18} u+1.472 e^{-18} v-2.055 e^{-17} w+1.295 e^{-17} e_{x}+$ $2.492 e^{-17} e_{y}+1.492 e^{-17} e_{z}-8.552 e^{-10}, F_{235}(x)=6.143 e^{-18} u-$ $6.037 e^{-18} v-3.121 e^{-17} w+2.936 e^{-18} e_{x}+2.769 e^{-17} e_{y}+$ $1.281 e^{-17} e_{z}+5.345 e^{-9}$, and $F_{236}(x)=1.147 e^{-18} u-$ $9.936 e^{-18} v-1.182 e^{-18} w+1.946 e^{-18} e_{x}+4.245 e^{-18} e_{y}+$ $2.831 e^{-18} e_{z}+0.2267$, and the degree of the polynomial $M_{i}(x)$ is set to be 0 to 1 (code for SOSTOOLS). When the degree is set to be different from what is set here, different $F_{i}(x)$ will be obtained accordingly.

Figures 5 and 6 depict the trajectory of the coaxial counter rotating helicopter dynamical system (59) under finite-time stabilizing control, which shows the validity and effectiveness of our proposed method.

\section{Conclusions}

Although numerous works have been published in recent years on the finite-time stability and stabilization of T-S fuzzy model, little attention has been paid to the investigation on 

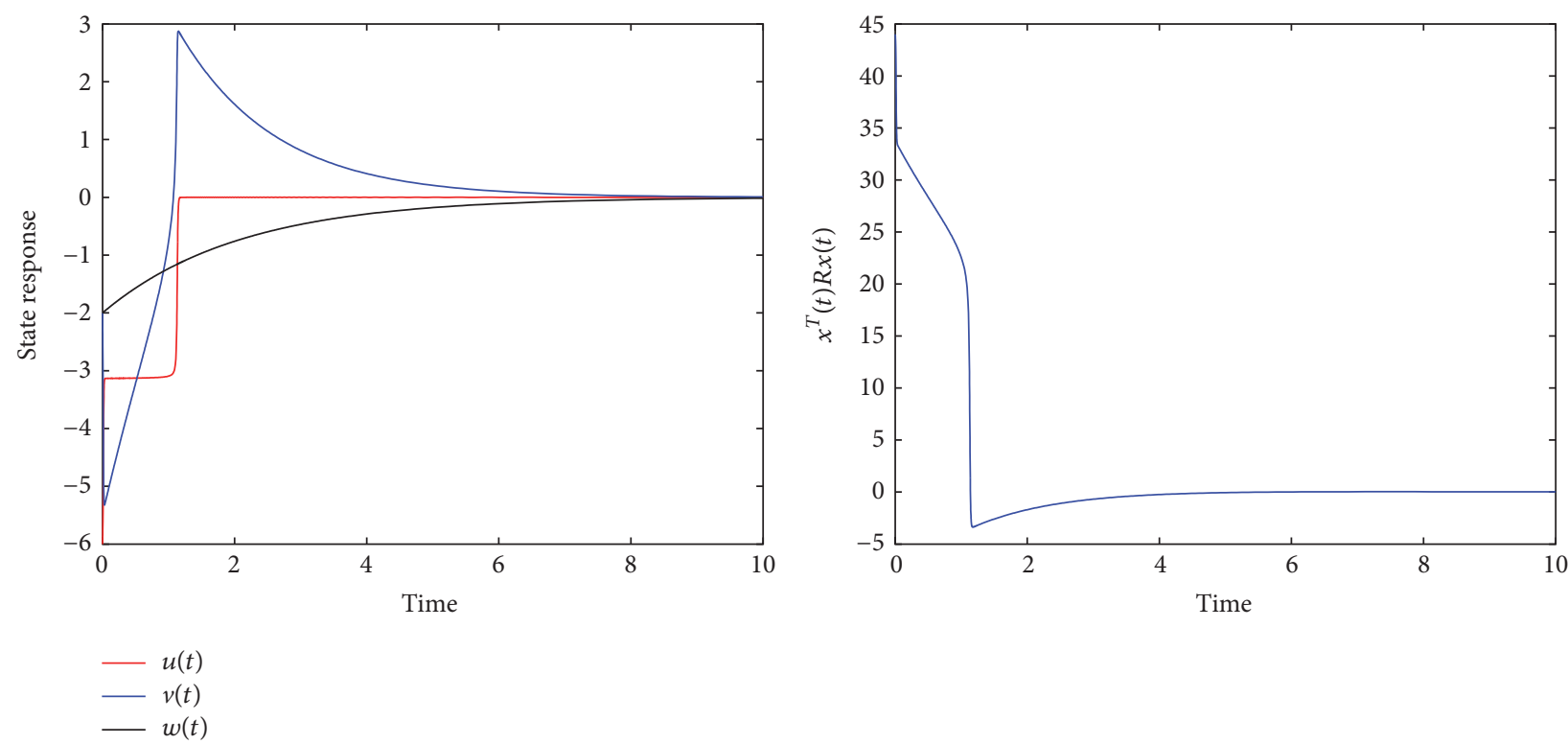

FIGURE 6: The state responses of polynomial fuzzy system (60) for the initial point $x(0)=\left[\begin{array}{lll}-6 & -2 & -2\end{array}\right]^{T}$.

the problem of finite-time stability analysis and synthesis method for continuous-time polynomial fuzzy system. The proposed results in this paper fill in this gap by using the polynomial Lyapunov functional approach and the SOS theory. Three illustrative examples have been provided to demonstrate the validity and effectiveness of the proposed results. In the future, we will consider the similar problem for the discrete-time polynomial fuzzy system and the polynomial fuzzy model with time delays or (and) uncertainties [17].

\section{Conflicts of Interest}

The authors declare that there are no conflicts of interest.

\section{Acknowledgments}

This work is supported by Natural Science Foundation of Jiangsu Province of China under Grant no. BK20161126.

\section{References}

[1] K. Tanaka and H. O. Wang, Fuzzy Control Systems Design and Analysis: A Linear Matrix Inequality Approach, Wiley, Hoboken, NJ, USA, 2001.

[2] H. Zhang and D. Liu, Fuzzy Modeling and Fuzzy Control, Control Engineering, Birkhäuser, Boston, Mass, USA, 2006.

[3] H. Zhang, S. Lun, and D. Liu, "Fuzzy $H_{\infty}$ filter design for a class of nonlinear discrete-time systems with multiple time delays," IEEE Transactions on Fuzzy Systems, vol. 15, no. 3, pp. 453-469, 2007.

[4] F. Yang and H. Zhang, "T-S model-based relaxed reliable stabilization of networked control systems with time-varying delays under variable sampling," International Journal of Fuzzy Systems, vol. 13, no. 4, pp. 260-269, 2011.
[5] X. Zhao, L. Zhang, P. Shi, and H. R. Karimi, "Novel stability criteria for T - S fuzzy systems," IEEE Transactions on Fuzzy Systems, vol. 22, no. 2, pp. 313-323, 2014.

[6] L. Wu, X. Su, P. Shi, and J. Qiu, "A new approach to stability analysis and stabilization of discrete-time T-S fuzzy timevarying delay systems," IEEE Transactions on Systems, Man, and Cybernetics, Part B: Cybernetics, vol. 41, no. 1, pp. 273-286, 2011.

[7] L. A. Mozelli, R. M. Palhares, F. O. Souza, and E. M. A. M. Mendes, "Reducing conservativeness in recent stability conditions of TS fuzzy systems," Automatica, vol. 45, no. 6, pp. 1580-1583, 2009.

[8] H. D. Choi, C. K. Ahn, P. Shi, L. Wu, and M. T. Lim, "Dynamic Output-Feedback Dissipative Control for T-S Fuzzy Systems With Time-Varying Input Delay and Output Constraints," IEEE Transactions on Fuzzy Systems, vol. 25, no. 3, pp. 511-526, 2017.

[9] Q. Zhou, H. Li, C. Wu, L. Wang, and C. K. Ahn, "Adaptive fuzzy control of nonlinear systems with unmodeled dynamics and input saturation using small-gain approach," IEEE Transactions on Systems, Man, and Cybernetics: Systems, vol. 47, no. 8, pp. 1979-1989, 2017.

[10] K. Tanaka, H. Yoshida, H. Ohtake, and H. O. Wang, "Stabilization of polynomial fuzzy systems via a sum of squares approach," in Proceedings of the 2007 IEEE 22nd International Symposium on Intelligent Control, ISIC 2007, pp. 160-165, Singapore, October 2007.

[11] K. Tanaka, H. Ohtake, T. Seo, M. Tanaka, and H. O. Wang, "Polynomial fuzzy observer designs: A sum-of-squares approach," IEEE Transactions on Systems, Man, and Cybernetics, Part B: Cybernetics, vol. 42, no. 5, pp. 1330-1342, 2012.

[12] K. Tanaka, H. Yoshida, H. Ohtake, and H. O. Wang, "A sum of squares approach to stability analysis of polynomial fuzzy systems," in Proceedings of the 2007 American Control Conference, ACC, pp. 4071-4076, New York, NY, USA, July 2007.

[13] K. Tanaka, H. Ohtake, and H. O. Wang, "Guaranteed cost control of polynomial fuzzy systems via a sum of squares approach," IEEE Transactions on Systems, Man, and Cybernetics, Part B: Cybernetics, vol. 39, no. 2, pp. 561-567, 2009. 
[14] K. Tanaka, H. Yoshida, H. Ohtake, and H. O. Wang, "A sum-ofsquares approach to modeling and control of nonlinear dynamical systems with polynomial fuzzy systems," IEEE Transactions on Fuzzy Systems, vol. 17, no. 4, pp. 911-922, 2009.

[15] K. Guelton, N. Manamanni, C.-C. Duong, and D. L. KoumbaEmianiwe, "Sum-of-squares stability analysis of Takagi-Sugeno systems based on multiple polynomial Lyapunov functions," International Journal of Fuzzy Systems, vol. 15, no. 1, pp. 1-8, 2013.

[16] S. Prajna, A. Papachristodoulou, P. Seiler, and P. Parrilo, SOSTOOLS: Sum of Squares Optimization Toolbox for MATLAB, version 3.01, California Institute of Technology, Pasadena, CA, USA, 2016.

[17] K. Tanaka, M. Tanaka, Y.-J. Chen, and H. O. Wang, "A new sumof-squares design framework for robust control of polynomial fuzzy systems with uncertainties," IEEE Transactions on Fuzzy Systems, vol. 24, no. 1, pp. 94-110, 2016.

[18] M. Narimani and H. K. Lam, "SOS-based stability analysis of polynomial fuzzy-model-based control systems via polynomial membership functions," IEEE Transactions on Fuzzy Systems, vol. 18, no. 5, pp. 862-871, 2010.

[19] H. K. Lam, "Polynomial fuzzy-model-based control systems: stability analysis via piecewise-linear membership functions," IEEE Transactions on Fuzzy Systems, vol. 19, no. 3, pp. 588-593, 2011.

[20] H. K. Lam, M. Narimani, H. Li, and H. Liu, "Stability analysis of polynomial-fuzzy-model-based control systems using switching polynomial lyapunov function," IEEE Transactions on Fuzzy Systems, vol. 21, no. 5, pp. 800-813, 2013.

[21] J. L. Pitarch, A. Sala, and C. V. Ariño, “Closed-form estimates of the domain of attraction for nonlinear systems via fuzzypolynomial models," IEEE Transactions on Cybernetics, vol. 44, no. 4, pp. 526-538, 2014.

[22] Z. Chen, B. Zhang, H. Li, and J. Yu, "Tracking control for polynomial fuzzy networked systems with repeated scalar nonlinearities," Neurocomputing, vol. 171, pp. 185-193, 2016.

[23] Y. Wang, H. Zhang, Y. Wang, and J. Zhang, "Stability analysis and controller design of discrete-time polynomial fuzzy timevarying delay systems," Journal of The Franklin Institute, vol. 352, no. 12, pp. 5661-5685, 2015.

[24] F. Amato, M. Ariola, and C. Cosentino, "Finite-time stabilization via dynamic output feedback," Automatica, vol. 42, no. 2, pp. 337-342, 2006.

[25] F. Amato, M. Ariola, and P. Dorato, "Finite-time control of linear systems subject to parametric uncertainties and disturbances," Automatica, vol. 37, no. 9, pp. 1459-1463, 2001.

[26] S. He and F. Liu, "Finite-time $H_{\infty}$ fuzzy control of nonlinear jump systems with time delays via dynamic observer-based state feedback," IEEE Transactions on Fuzzy Systems, vol. 20, no. 4, pp. 605-614, 2012.

[27] D. Yang and K.-Y. Cai, "Finite-time quantized guaranteed cost fuzzy control for continuous-time nonlinear systems," Expert Systems with Applications, vol. 37, no. 10, pp. 6963-6967, 2010.

[28] H. Shen, J. H. Park, and Z.-G. Wu, "Finite-time reliable control for Takagi-Sugeno fuzzy systems with actuator faults," IET Control Theory \& Applications, vol. 8, no. 9, pp. 688-696, 2014.

[29] C. Zheng, J. Cao, M. Hu, and X. Fan, "Finite-time stabilisation for discrete-time T-S fuzzy model system with channel fading and two types of parametric uncertainty," International Journal of Systems Science, vol. 48, no. 1, pp. 34-42, 2017.
[30] S. Prajna, A. Papachristodoulou, and F. Wu, "Nonlinear control synthesis by sum of squares optimization: A Lyapunov-based approach," in Proceedings of the 2004 5th Asian Control Conference, pp. 157-165, Melbourne, Victoria, Australia, July 2004.

[31] K. Tanaka, T. Komatsu, H. Ohtake, and H. O. Wang, "Micro helicopter control: LMI approach vs SOS approach," in Proceedings of the 2008 IEEE International Conference on Fuzzy Systems, FUZZ 2008, pp. 347-353, Hong Kong, China, June 2008. 


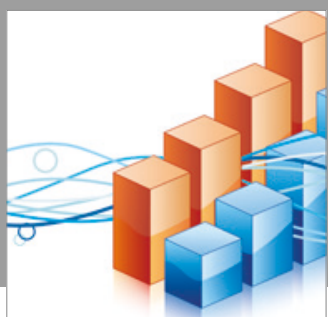

Advances in

Operations Research

vatersals

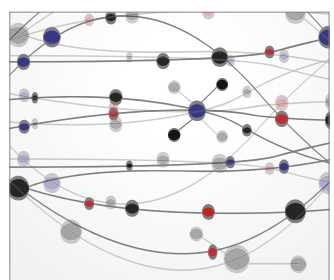

\section{The Scientific} World Journal
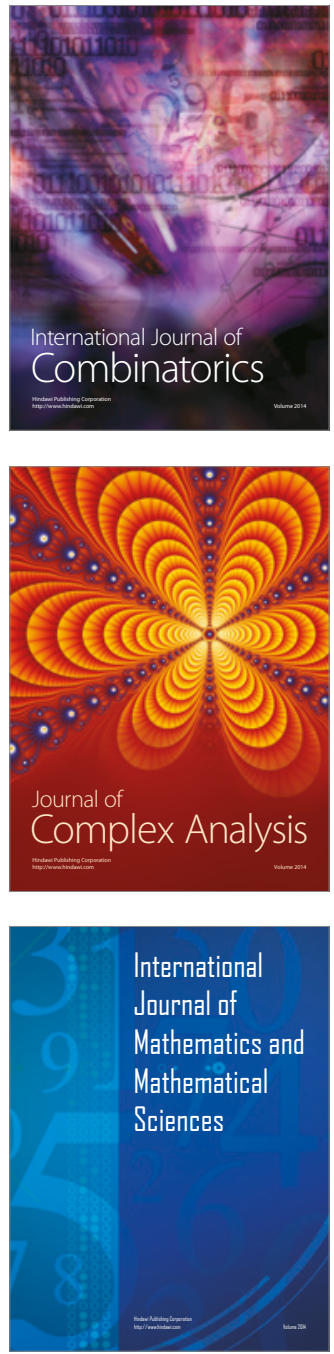
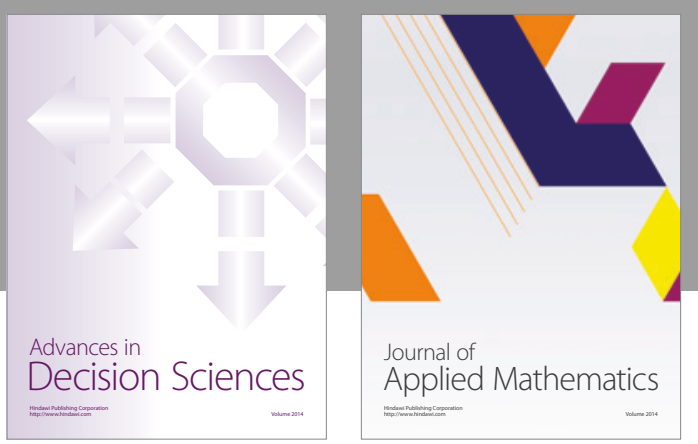

Algebra

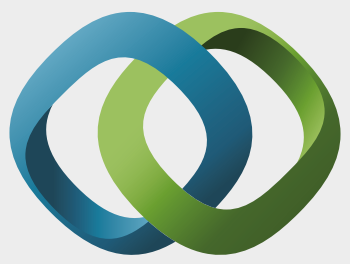

\section{Hindawi}

Submit your manuscripts at

https://www.hindawi.com
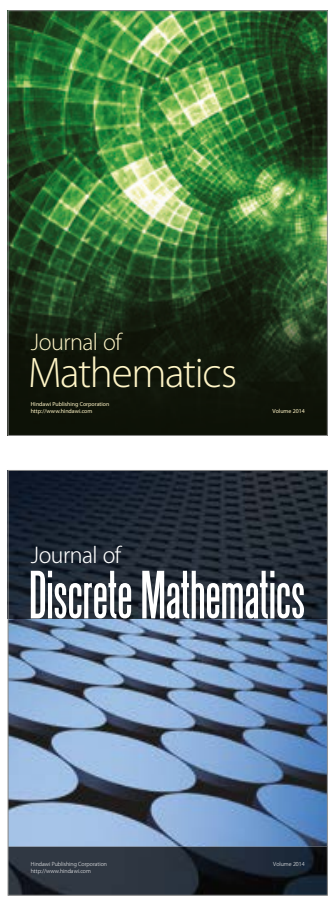

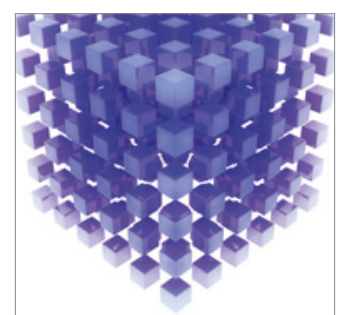

Mathematical Problems in Engineering
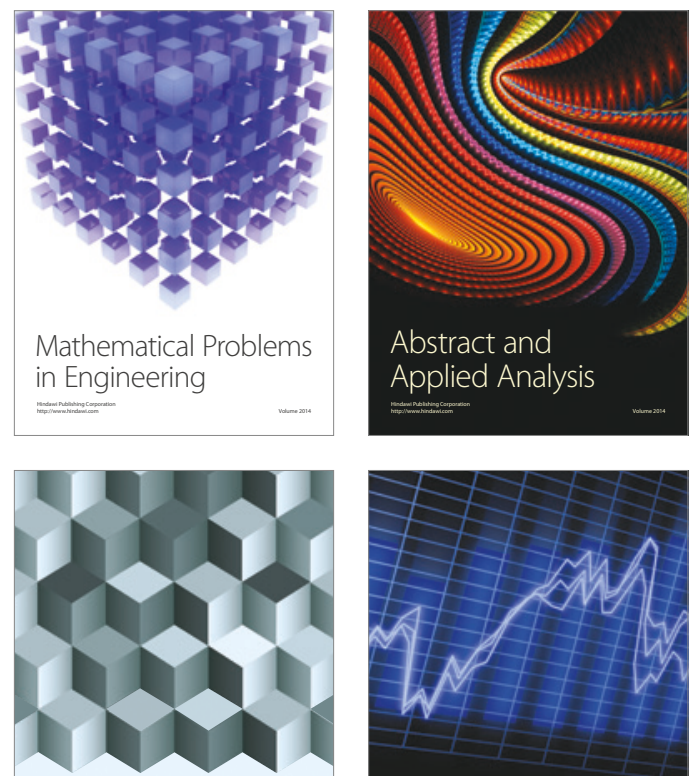

Journal of

Function Spaces

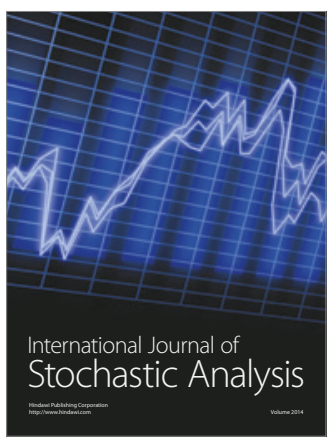

Probability and Statistics
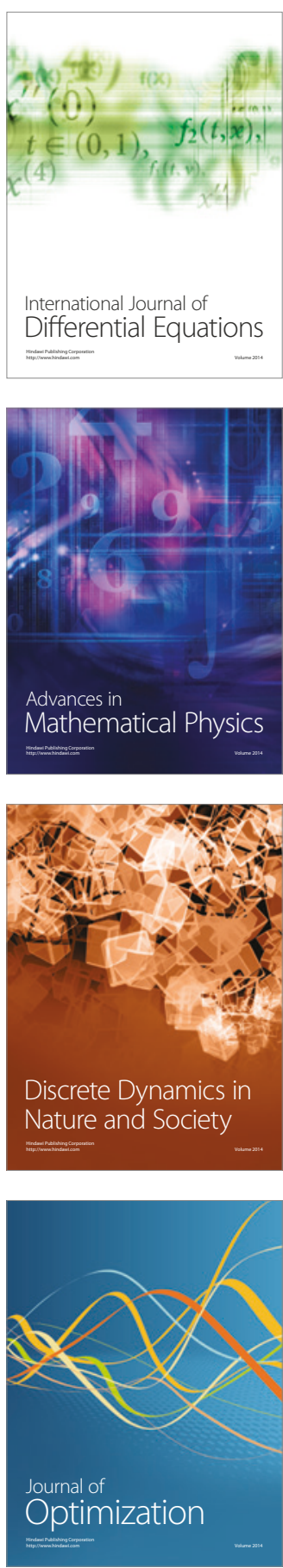